\title{
KINETIC STUDIES OF ANOMALOUS TRANSPORT
}

\author{
W. M. Tang \\ Princeton Plasma Physics Laboratory, Princeton University \\ Princeton, New Jersey 08543, USA.
}

\begin{abstract}
Progress in achieving a physics-based understanding of anomalous transport in toroidal systems has come in large part from investigations based on the proposition that low frequency electrostatic microinstabilities are dominant in the bulk ("confinement") region of these plasmas. Although the presence here of drift-type modes dependent on trapped particle and ion temperature gradient driven effects appears to be consistent with a number of important observed confinement trends, conventional estimates for these instabilites cannot account for the strong current $\left(I_{p}\right)$ and /or $q$-scaling frequently found in empirically deduced global energy confinement times for auxiliary-heated discharges. The present paper deals with both linear and nonlinear physics features, ignored in simpler estimates, which could introduce an appreciable local dependence on current. It is also pointed out that while the thermal flux characteristics of drift modes have justifiably been the focus of experimental studies assessing their relevance, other transport properties associated with these microinstabilities should additionally be examined. Accordingly, the present paper provides sstimates and discusses the significance of anomalous energy exchange between ions and electrons when fluctuations are present.
\end{abstract}




\section{Introduction}

It has been pointed out in a number of papers ${ }^{1-3}$ that the presence of electrostatic microturbulence due to drift-type modes is consistent with some important confinement trends observed in tokamak plasmas. For example, in most auxiliary heated tokamaks the global energy confinement time $\left(\tau_{E}\right)$ has been observed to anomalously degrade with power $(P)$ as $\tau_{E} \propto P^{-0.5}$. This trend ${ }^{4}$ correlates reasonably well with predictions from theories for collisionless drift-type instabilities driven by trapped electrons and/or by ion temperature gradients. Specifically, application of Connor-Taylor ${ }^{5}$ invariance transformation arguments to the nonlinear gyrokinetic equation together with the heat transport equation leads to the result that the local diffusivity, $\chi$, must scale as

$$
\chi \propto \frac{T^{3 / 2}}{B^{2} a}
$$

for all collisionless low frequency electrostatic instabilities. ${ }^{6}$ From simple power balance,

$$
\chi \propto \frac{P}{n T R}
$$

Combining Eqs. (1) and (2) gives

$$
T \propto\left(\frac{P a B^{2}}{n R}\right)^{0.4}
$$

Since the confinement time scales as $\tau_{E} \propto R a^{2} n T / P$, Eq. (3) leads to

$$
\tau_{E} \propto P^{-0.6} B^{0.8}
$$

Although Eq. (4) is in good agreement with the important power degradation trend observed, it fails to exhibit the strong scaling with current $\left(I_{p}\right)$ and the absence of any significant dependence on toroidal field $(B)$ found in the global energy confinement time empirically deduced from a large data base of auxiliary heated tokamak discharges; ${ }^{4}$ i.e.,

$$
\left(\tau_{E}\right)_{\text {global }}^{\text {empirical }} \propto P^{-0.5} I_{p}^{1.0} .
$$


This discrepancy could be resolved if the local diffusivity contained a significant dependence on the safety factor, $q \equiv r^{2} B / I_{p} R$. Specifically, since the Connor-Taylor similarity tranformation arguments cannot generally deal with dependencies on dimensionless parameters of this type, it is possible to consider a diffusivity of the form,

$$
\chi \propto T^{3 / 2} q^{x} / B^{2} a .
$$

Repearing the preceding simple arguments leading to Eq. (4) then yields (focusing only on the $B$ vs. $I_{p}$ issue),

$$
\tau_{E} \propto P^{-0.6} B^{0.8}\left(I_{p} / B\right)^{0.4 x} .
$$

Hence, reasonable agreement with Eq. (5) would result for an appropriate value of $x$; e.g., if $x=2.5$, then Eq. (7) would give $\tau_{E} \propto P^{-0.6} I_{p}^{1.0} B^{-0.2}$. It, therefore, remains to be demonstrated that a sufficiently strong dependence on $q$ can be justified on the basis of a proper kinetic analysis of the relevant microinstabilities. Indeed, transport models based on relatively simple pictures of drift-type modes have needed to invoke either $\mathrm{em}$ | irical $q$-dependent profile constraints ${ }^{2,7}$ or ad hoc $q$-dependent edge degradation processes ${ }^{1,3}$ in order to reasonably reproduce experimentally observed confinement results.

Since most of the previous simple linear and nonlinear $\left(\chi \propto \gamma / k_{\perp}^{2}\right)$ estimates for drift-type microinstabilities have yielded no obvious local current and/or $q$-dependences, the present paper deals with kinetic studies carefully examining effects which might do so. The focus of these in estigations will be on a proper linear and nonlinear (weak turbulence) analysis of collisionless trapped electron modes. ${ }^{8}$ In addition to the fact that conditions required for their presence are readily satisfied in many auxiliary heated large tokamak plasmas, the properties of these instabilities make them amenable to a systematic appiication of weak turbulence nonlinear kinetic analysis.

\section{Linear Analysis}

Collisionless trapped electron modes (CTEM) have long been recognized as a potentially important class of instabilities influencing confinement properties in high temperature toroidal plasmas. ${ }^{8}$ These are basically electron drift waves in a toroidal system 
with characteristic mode frequencies in the range, $\omega_{b i}, k_{\|} v_{T i}<\omega<\omega_{b e}, k_{\|} v_{T_{e}}$; i.e., falling between the bounce and transit frequencies of the ions and the electrons respectively. In addition to the banana regime collisionality criterion, $\nu_{* e} \equiv \nu_{e i} / \epsilon \omega_{b e}<1$, the effective electron-ion collision frequency, $\nu_{e i} / \epsilon$, is required to be less than the eigenmode frequency, $\omega$. Since $\omega \sim \omega_{\text {*e }}$, this collisionality criterion in terms of $\nu_{* e}$ becomes,

$$
\nu_{* e}<k_{\theta} \rho_{\theta}\left(m_{e} / M_{i}\right)^{1 / 2} q R / L_{n}
$$

where $\omega_{* e} \equiv k_{\theta} \rho_{s} c_{s} / L_{n}$ is the electron diamagnetic drift frequency, $c_{s}$ is the sound speed, $k_{\theta}$ is the azimuthal wave number, $\rho_{s} \equiv c_{s} / \Omega_{i}, \Omega_{i}$ is the ion cyclotron frequency, and $L_{n}$ is the density gradient scale length. This condition is readily satisfied in the confinement region of many large tokamak auxiliary heated plasmas. Fur example, in the TFTR supershot discharges, ${ }^{9}$ typical parameters in this region would be $\nu_{\text {*e }} \sim 0.02$, $q \sim 3 / 2, R / a \sim 3, L_{n} \sim a / 3$, so that for a deuterium plasma, Eq. (8) would reduce to the wave number requirement, $k_{\theta} \rho_{\mathrm{o}}>0.1$.

At moderate wavelengths, $0.1<k_{\theta} \rho_{s}<1$, the dominant destabilization mechanism for the collisionless electrostatic modes of interest are the precession resonances between the drift waves and the unfavorably drifting trapped electrons. ${ }^{8}$ This resonance mechanism is analogous to the energetic trapped-ion precession resonances accounting for the experimentally observed "fishbone" losses in neutral-beam heated tokamaks. ${ }^{10}$ At the simplest level, the local dispersion relation for the CTEM can be approximated by

$$
1+\tau-\left(\tau+\frac{\omega_{\text {*e }}}{\omega}\right) \Gamma_{0}-\eta_{i} \frac{\omega_{\text {*e }}}{\omega} b_{i}\left(\Gamma_{1}-\Gamma_{0}\right)=\epsilon^{1 / 2}\left\langle\frac{\omega-\omega_{* e}\left[1+\eta_{e}\left(E / T_{e}-3 / 2\right)\right]}{\omega-\bar{\omega}_{d e}^{(0)}}\right\rangle_{E}
$$

with $\Gamma_{n} \equiv I_{n}\left(b_{i}\right) \exp \left(-b_{i}\right), b_{i} \equiv k_{\theta}^{2} \rho_{i}^{2} / 2, \tau \equiv T_{c} / T_{i}, \eta_{i} \equiv d \ln T_{i} / d \ln n_{i}, \epsilon^{1 / 2}$ being the fraction of trapped electrons, the angular brackets denoting an energy average over a Maxwellian distribution, and the orbit-averaged trapped electron precession frequency given by

$$
\bar{\omega}_{d e}^{(0)} \equiv \omega_{* e} \frac{L_{n e}}{R} \frac{E}{T_{e}} G .
$$

In most previous studies the factor $G$ is assumed for simplicity to be a constant near unity. For $\eta_{i} \lesssim 1$ to $2, \tau \lesssim 1$, and $k_{\theta} p_{s}<1$, the left side of Eq. (9) just reduces to the 
usual electron drift wave result while the right side can be perturbatively evaluated to give the growth rate $(\gamma)$. The resonant destabilization comes from $\operatorname{Im}\left[\left(1 /\left(\omega-\bar{\omega}_{d e}^{(0)}\right)\right]\right.$ and leads to

$$
\gamma \propto x^{3 / 2}(x-3 / 2) \exp (-x)
$$

with $x \equiv\left(E / T_{e}\right)_{\text {res }} \simeq R / L_{n} \mathcal{G}_{\text {ave }}$ being the normalized value of the resonant energy. This conventional estimate leads to no significant current and/or $q$-dependence in the linear properties of the CTEM, when $G_{a v e}$ is simply assumed to be a constant of order unity.

Regarding the linear aspects of the present analysis, attention is focused on the fact that current dependencies $\left(I_{p}\right)$ can be introduced via the magnetic shear dependence of the factor $G$ in the trapped-electron precession drifts and via the $q$-dependence of the stabilizing ion Landau damping effects ignored in most conventional estimates for the CTEM. The first point is illustrated in Fig. 1 and Fig. 2. On Fig. 1 the factor $G(\kappa, \hat{s})$ is plotted as a function of the velocity-space pitch angle variable, $\kappa$, for various representative equilibrium scenarios where the total plasma current (and, therefore, $\left.q_{a} \equiv a^{2} B / I_{p} R\right)$ is changed. When averaged over $\kappa$, it is obvious that the high current (low $q_{a}$ and corresponding low $\hat{s} \equiv r q^{\prime}, q$ ) cases give the smallest values for $G_{a v e}$. The direct connection between $\hat{s}$ and $G_{a v e}$ is more explicitly shown in Fig. 2 for simpler analytic equilibria. The main point made here is that the strength of the CTEM depends sensitively on the factor $G$ which in turn can have a significant dependence on current via the shear parameter $\hat{s}$. With respect to ion Landau damping, the effect obviously becomes stronger as the ion bounce $\left(\omega_{b i}\right)$ and transit frequencies $\left(\omega_{t i}\right)$ approach the eigenmode frequency $(\omega)$. This favorable influence on $\omega$ clearly improves as $I_{p}$ increases since $\omega_{t i}$ and $\omega_{b i}$ (both $\propto 1 / q$ ) would correspondingly increase toward $\omega$.

Up to this point, the arguments given for the presence of a current dependence in CTEM have been primarily qualitative. In order to demonstrate this quantitatively, comprehensive linear eigenmode calculations have been numerically carried out. The complex code ${ }^{11}$ employed provices solutions to the appropriate toroidal eigenmode equation without any frequency expansions. Thus, the precession resonances as well as the ion bounce and transit resonances are all evaluated without approximations. 


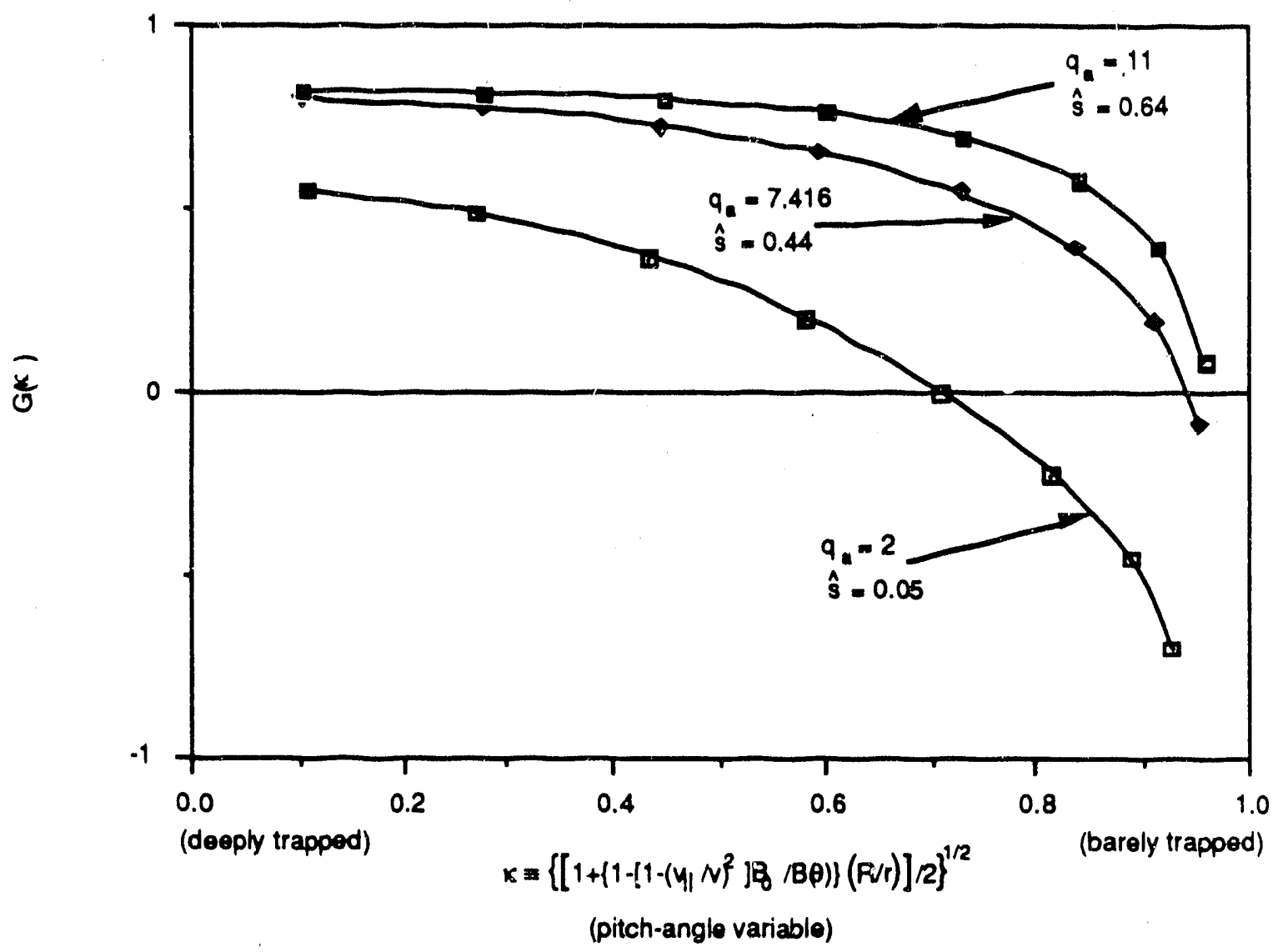

Figure 1: Plot of the $G$-factor from the trapped-particle precession frequency expression given in Eq. (10) as a function of the velocity-space pitch-angle variable. This figure illustrates that at higher currert (corresponding to typically smaller values of $q_{a}$ and $\hat{s}$ ), the $G$-factor is also smaller. 


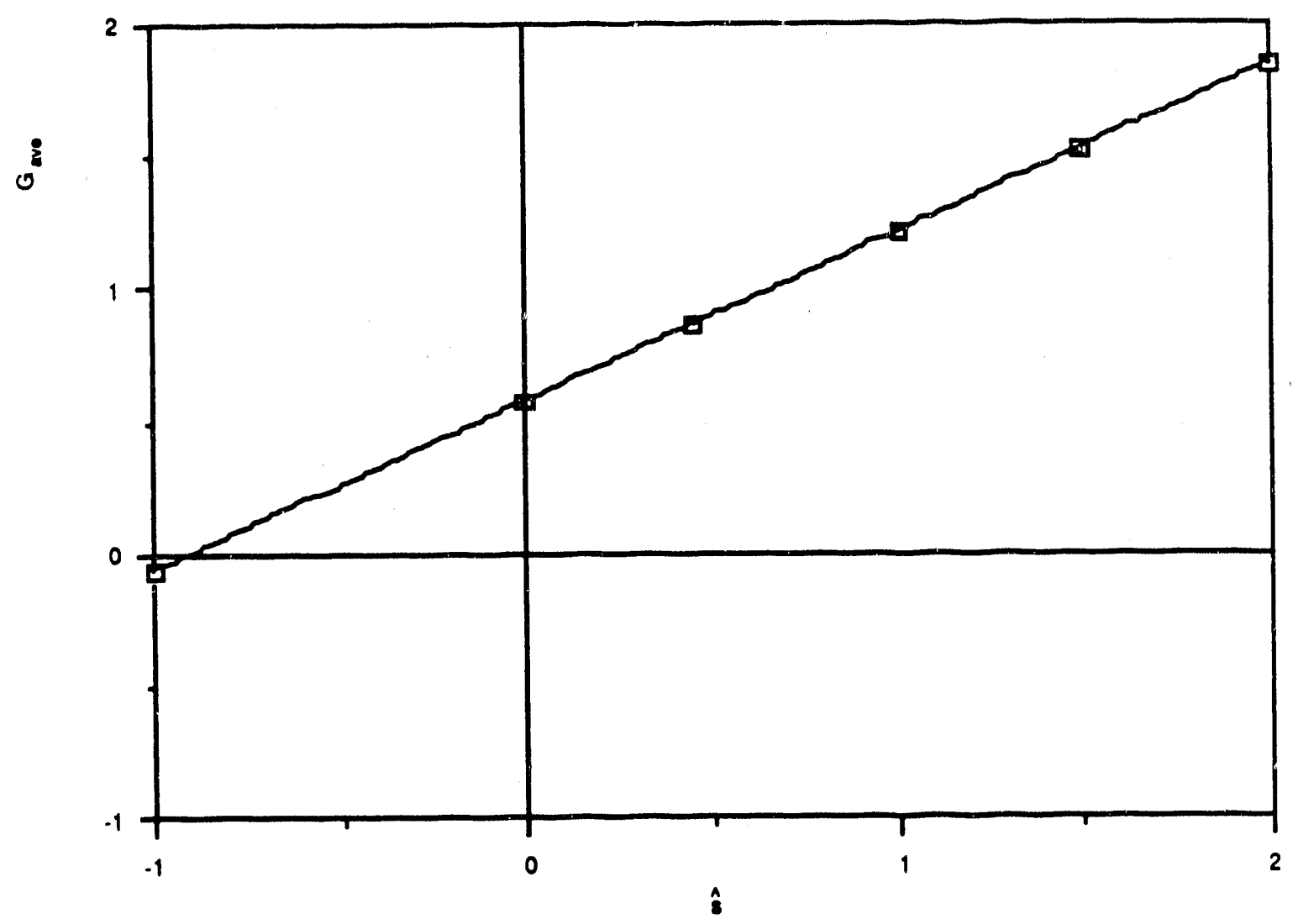

Figure 2: Plot of the $G$-factor averaged over pitch-angle $\left(G_{a v e}\right)$ for simple analytic equilibria as a function of $\hat{s}$. 
The usual ballooning formalism is employed, so that the calculations are local to a single, chosen magnetic surface. This stability analysis is interfaced with self-consistent numerically computed MHD equilibria. In studying the possible dependence on current, the $q(r)$ profile is varied by changing $q(a)$ (i.e., changing the total plasma current) at fixed $q(o)$ with intermediate $q(r)$ values scaling as

$$
q_{\text {new }}(r)=\frac{\left[q_{\text {new }}(a)-q(o)\right]}{\left[q_{\text {old }}(a)-q(o)\right]}\left[q_{\text {old }}(r)-q(o)\right]+q(o) .
$$

A numerical MHD equilibrium is recomputed for each new $q(r)$ profile, and the corresponding growth rate, $\gamma$, is then maximized over $k_{\theta}$. Results from these calculations indicate a dependence of the linear growth rate on $q(a)$ scaling roughly as $\gamma \propto[q(a)]^{\alpha}$ with $\alpha \sim 0.5$ to 1.5 for values of $q(a)$ below 7. For values of $q(a)>7$, the dependence becomes rather weak. These trends are illustrated in Fig. 3 for a representative case. Results here are consistent with the preceding qualitative arguments given for the precession drifts and for ion Landau damping.

\section{Weak Turbulence Nonlirear Analysis}

In order to assess the impact of CTEM on confinement, it is necessary to estimate the saturation level of these instabilites and the associated anomalous particle and thermal fluxes. At the simplest level, this has been done by using heuristic mixing length approximations for saturation (e.g., $\left.\left|e \phi / T_{e}\right| \sim 1 / k_{\perp} L_{v}\right)$ and heuristic random walk type arguments for the effective anomalous diffusivities (e.g., $\chi_{\text {eff }} \sim \gamma / k_{\perp}^{2}$ ). When coupled with conventional simple estimates for $\gamma$, this approach leads to no significant dependence of transport on current. Even if the improved detailed linear theory results described in Sec. II were invoked, the associated scaling for typical cases would be no stronger than $\chi_{\text {eff }} \propto[q(a)]^{\alpha}$ with $\alpha \sim 0.5$ to 1.5 for values of $q(a)$ below 7 . Howevir, since the properties of the CTEM make them amenable to a systematic application of weak turbulence nonlinear analysis, ${ }^{13}$ it is appropriate to focus attention on this more rigorous approach.

In this section a description is given of the principal features and results of a weak turbulence theory for the CTEM in toroidal geometry. ${ }^{14}$ The usual weak turbulence 


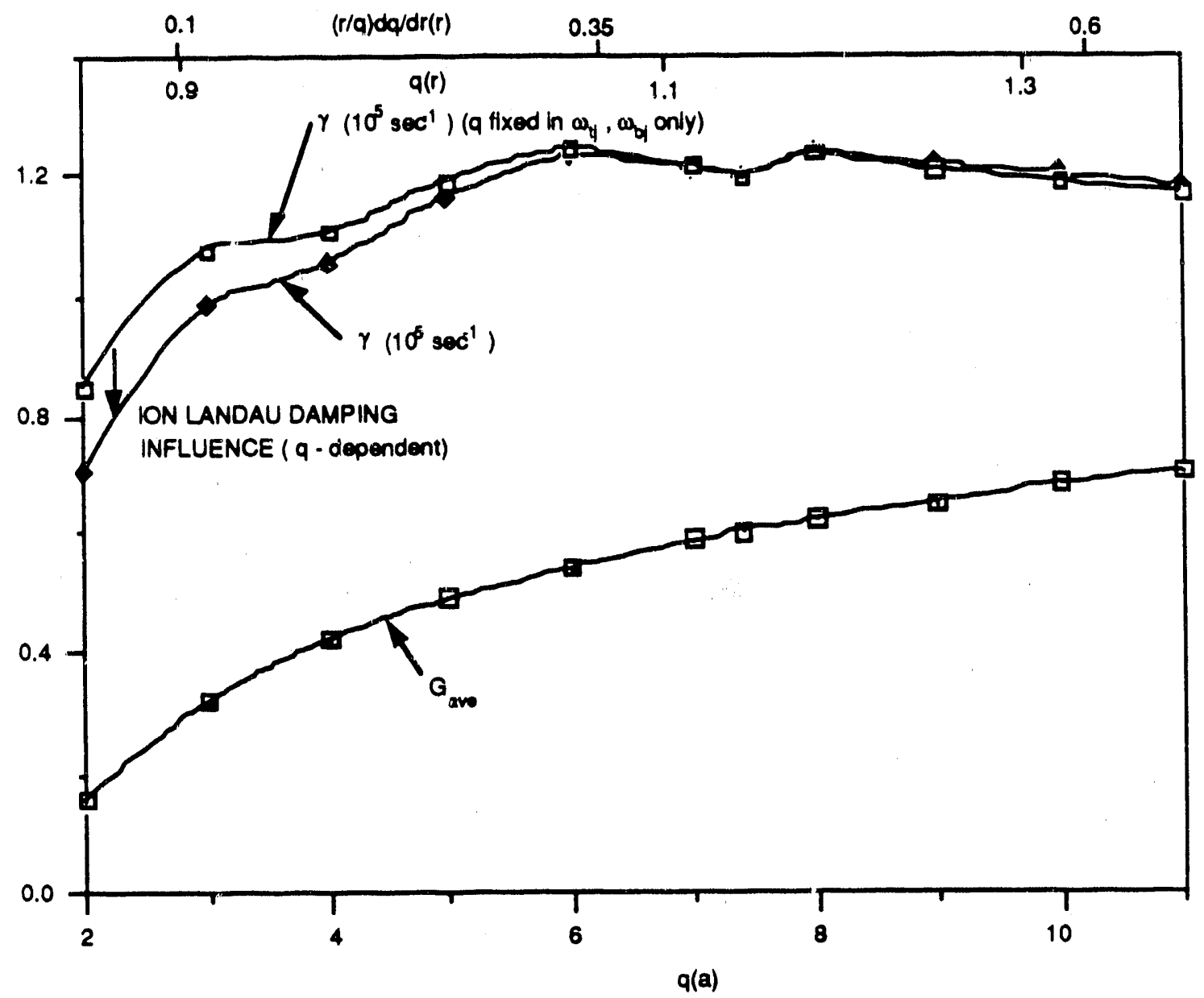

Figure 3: Plot of linear growth rate $(\gamma)$ as a function of $q_{a}$ for representative TFTR parameters in the collisionless electrostatic limit. The lower $\gamma$-curve shows the influence of ion Landau damping. The $G_{\text {ave }}$ factor for these specific numerical equilibria is also plotted. 
ordering $^{13}\left(|e \phi / T| \sim\left|\gamma / \omega_{r}\right| \sim\left|\Delta \omega_{N L} / \omega_{r}\right| \ll 1\right.$ with $\Delta \omega_{N L}$ being the nonlinear frequency spread) is invoked, and a simple model of the radial eigenmode structure is adopted with $\phi \propto \exp \left[-\left(x^{2} / 2(\Delta x)^{2}\right)\right]$, where $\Delta x \equiv 1 / k_{\theta} \hat{s}$ represents the spacing between rational surfaces and $x \equiv r-r_{0}$ is the local radial coordinate. With regard to the basic equations analyzed, the ion response is governed by the nonlinear gyrokinetic equation:

$$
\left(\partial_{t}+i \omega_{d i}+v_{\|} \hat{b} \cdot \nabla\right) g+i \tau\left[\omega-\omega_{* i}\left\{1+\left(u^{2}-3\right) \eta_{i} / 2\right\}\right] \Phi J_{0} F_{m}=\nabla \Phi J_{0} \times \hat{b} \cdot \nabla g
$$

where $g$ is the nonadiabatic part of the perturbed distribution function, $\hat{b} \equiv \mathbf{B} / B$, $\Phi \equiv e \phi / T_{e}, u^{2}=M_{i} v^{2} / T_{i}, J_{0}\left(k_{\perp} \rho_{i}\right)$ is a Bessel function, and $F_{m}$ is a Maxwellian. The nonlinear bounce-averaged drift-kinetic equation determines the trapped electron response,

$$
\left(\partial_{t}+i \bar{\omega}_{d e}^{(0)}\right) h-i\left[\omega-\omega_{* e}\left\{1+\left(E / T_{e}-3 / 2\right) \eta_{e}\right\}\right]<\Phi>F_{M}=\nabla<\Phi>\times \hat{b} \cdot \nabla h
$$

where $h$ is the nonadiabatic perturbed distribution for trapped electrons and $\langle\Phi\rangle$ is the bounce-average of $\Phi$. Untrapped electrons are simply taken to be represented by the usual Boltzmann response.

The nonlinear evolution of the spectral intensity, $I(\mathbf{k}) \equiv\left|\boldsymbol{\Phi}_{\mathbf{k}}\right|^{2}$, is determined by the appropriate wave kinetic equation. This is obtained by a standard application of weak turbulence expansion procedures ${ }^{13}$ to Eqs. (13) and (14) with the nonlinear terms (on the right side of each equation) treated perturbatively. To leading (first) order, the usual linear response of a test mode $(\mathbf{k})$ is obtained. The nonlinear interaction of the test mode $(\mathbf{k})$ with background modes $\left(\mathbf{k}^{\prime}\right)$ to produce driven modes $\left(\mathbf{k}^{\prime \prime}\right)$ is generated at second order. At third order, the nonlinear interactions of $\mathbf{k}^{\prime}$ and $\mathbf{k}^{\prime \prime}$ produce the back reaction on the test mode $(\mathbf{k})$. Application of the quasineutrality condition on both the linear and nonlinear responses yields

$$
(1+\tau) \Phi_{\mathbf{k}}+\int d^{3} v\left(h_{\mathbf{k}}^{(1)}-g_{\mathbf{k}}^{(1)} J_{0}\right)=-\int d^{3} v\left(h_{\mathbf{k}}^{(3)}-g_{\mathbf{k}}^{(3)} J_{0}\right) .
$$

The imaginary part of this equation is just the wave kinetic equation of interest.

Details of the preceding derivation alre presented in Ref. 14. As pointed out there, the inherent dispersive character of the relevant eigenmode frequency renders the 3wave-type nonlinear interactions insignificant. Specifically, $\omega$ is a dispersive function 
of $k_{\theta}$ due to the finite ion gyroradius effects evident in Eq. (9). The dominant nonlinear process involves wave-particle-wave interactions; i.e., $\omega^{\prime}-\omega=\left(\mathbf{k}^{\prime}-\mathbf{k}\right) \cdot \mathbf{v}$ with $\omega^{\prime \prime}=\omega^{\prime}-\omega$ and $k^{\prime \prime}=k^{\prime}-\mathbf{k}$. For the ions, the nonlinear contributions from the right side of Eq. (13) leads to the nonlinear ion Landau damping (ion Compton scattering) which transfers fluctuation energy to the longer wavelength modes in the spectrum. The trapped electron scattering, generated by the nonlinear term on the right side of Eq. (14), accounts for a transfer of fluctuation energy to the shorter wavelenth modes. For the nonlinearly saturated state $(\partial / \partial t \rightarrow 0)$, the solution of the wave kinetic equation represents a balance between these two $\mathbf{E} \times \mathbf{B}$-type monlinear processes. In the longer wavelength regime $\left[\left(L_{n} / R\right)^{1 / 2}<k_{\theta} \rho_{s}<1\right]$, where most of the fluctuation energy resides, this ieads to the following expression for the radially averaged spectral intensity: ${ }^{14}$

$$
<I\left(k_{\theta}\right)>_{R}^{1 / 2}=\left|\left(\frac{\tilde{n}}{n}\right)_{\mathbf{k}}\right| \sim\left[\epsilon^{1 / 2} x^{7 / 2}\left(x-\frac{3}{2} \exp (-x)\right) \frac{G^{2} \tau}{\left[1+\frac{5}{4} \eta_{i}\right]} \frac{q^{3}}{\hat{s} k_{\theta} r_{0}}\right]^{1 / 2} \frac{1}{k_{\theta} L}
$$

with $\epsilon \equiv r_{0} / R, L \equiv\left(L_{n} L_{T_{e}}\right)^{1 / 2}$, and $x \equiv\left(E / T_{e}\right)_{\text {res }} \simeq R / L_{n} G$ [as in Eq. (11)]. For typical parameters, this result gives a smaller saturation amplitude than the familiar mixing length estimate. It is interesting to note that the appropriate length scale, $L \equiv\left(L_{n} L_{T e}\right)^{1 / 2}$, also differs from the conventional designation, $L=L_{n}$, found in most drift wave estimates of saturation. The present result is in fact more realistic since the instability of interest is obviously dependent on the driving free energy associated with both the density and the electron temperature gradients. With respect to the issue of curr int scaling, the presence of the factors $q, \dot{s}, G$, and $x$ in Eq. (16) clearly indicates a significant sensitivity to $I_{p}(r)$ in the saturated amplitude of the CTEM.

The fluctuation-induced anomalous fluxes associated with the CTEM can now be readily estimated using Eq. (16). For example, the particle flux is given by

$$
\Gamma=\Gamma_{e}=\operatorname{Re} \sum_{\mathbf{k}} \int d^{3} v\left(h_{k}^{(1)}+h_{k}^{(3)}\right) \frac{i k_{\theta} c}{B} \phi_{-k} .
$$

Since the effective anomalous diffusivities can themselves be functions of the density and temperaure gradients, it is convenient to express the anomalous fluxes as $\Gamma \equiv$ $-D^{\text {eff }} d n_{0} / d r$ and $Q_{j} \equiv-\chi^{\text {eff }} n_{0} d T_{j} / d r$. From the present weak turbulence analysis of 
the CTEM, this leads to

$$
\begin{aligned}
& D^{\text {eff }} \sim 10 \epsilon x^{5}\left(x-\frac{3}{2}\right) \exp (-2 x)\left(\frac{\eta_{e} G^{2}}{1+\frac{5}{4} \eta_{i}}\right) \tau\left(\frac{q}{\hat{s}}\right)^{2}\left(\frac{c T_{e}}{e B} \frac{\rho_{s}}{L_{T e}}\right) \\
& \chi_{e}^{\text {eff }} \sim \frac{x}{\eta_{e}} D^{\text {eff }},
\end{aligned}
$$

and

$$
\chi_{i}^{\text {eff }} \sim\left(\frac{2.75}{\eta_{i}}\right) \frac{\left(1+1.93 \eta_{i}\right)}{\left(1+\frac{5}{4} \eta_{i}\right)} D^{\text {eff }} .
$$

Hence, $\chi_{e}^{\text {eff }} \sim \chi_{i}^{\text {eff }} \gtrsim D^{\text {eff }}$ are all roughly the same order of magnitude. Also, the factor $\left(c T_{e} / e B\right)\left(\rho_{s} / L_{T e}\right) \sim T^{3 / 2} / B^{2} L_{T e}$ gives the so-called "gyro-reduced Bohm" scaling with $L_{T e}$ being the srecific gradient scale length. Regarding the important issue of local current scaling, note that Eq. (19) yields $\chi_{e}^{\text {eff }} \propto(q / \hat{s})^{2} F(q, \hat{s})$ with $F(q, \hat{s}) \sim$ $x^{6}(x-3 / 2) \exp (-2 x) G^{2} \propto G^{2}$. Typically, for $q(a)<5, G \propto q(a)$. Representative data from TFTR ${ }^{14}$ also indicates that at the half-radius, $(q / 3) \propto[q(a)]^{1.2}$. Hence, very roughly, $\chi_{e}^{\text {eff }} \propto[q(a)]^{2.2}$ for this specific example.

\section{Anomalous Energy Exchange from Fluctuations}

Although the particle and thermal flux properties of drift-type microinstabilities have justifiably been the focus of experimental studies assessing their relevance, other transport properties associated with these modes should additionally be examined. In particular, since electrostatic microturbulence is often cited as likely being responsible for anomalous thermal transport in tokamaks, it would be inconsistent to ignore the anomalous energy exchange between ions and electrons when fluctuations are present. This can be formally obtained from the appropriate moment of the Boltzmann equation and is generally expressed as ${ }^{15}$

$$
\dot{W}_{j}=-\left\langle\delta \mathbf{E}^{*} \cdot \delta \tilde{\mathbf{j}}_{j}>\right.
$$

where $\delta \tilde{J}_{j} \equiv e_{j} \int d^{3} v \mathbf{v} f_{j}$ and $f_{j}$ is the perturbed distribution function for species $j$. Using the continuity equation, this can be more conveniently expressed as

$$
\dot{W}_{j}=<\tilde{\phi} * \frac{\partial}{\partial t} \rho_{j}>=e_{j}<\tilde{\phi} * \frac{\partial}{\partial t} \tilde{n}_{j}>
$$


with $\delta \tilde{\mathbf{E}}^{*}=i \mathbf{k} \tilde{\phi} *$. Note that the adiabatic contribution to the perturbed density response cannoc contribute to energy exchange in the saturated stite since $\partial / \partial t\left(|\phi|^{2}\right)=0$. From power balance considerations, the total heat source for species $j$ is just $P_{j}-\dot{W}_{j}$ with $\sum_{j} \dot{W}_{j}=0$. So, $\dot{W}_{j}>0$ corresponds to "cooling" of species $j$ (i.e., a transfer of heat to the other species).

At the simplest level, application of standard quasilinear theory leadis to the following estimate for the anomalous energy exchange associated with the CTEM,

$$
\dot{W}_{e}^{\text {CTEM }} \simeq \frac{T_{e}}{L_{n}} \Gamma_{e}^{\text {CTEM }}\left[1-\frac{L_{n} G}{R}\left(1+\eta_{e}\right)\right] .
$$

Comparison with the dominant anomalous thermal conduction contribution to power balance yields

$$
\left(\frac{\dot{W}_{\mathrm{e}} a}{Q_{\mathrm{e}}}\right)^{\mathrm{CTEM}} \sim \frac{a}{R}\left[1-\frac{L_{\mathrm{n}} G}{R}\left(1+\eta_{\mathrm{e}}\right)\right] .
$$

Hence, for typical tokamak parameters, $\dot{W}_{e}$ could produce up to about a $20 \%$ effect.

\section{Concluding Comments}

In the kinetic studies of ansmalous transport presented in this paper, the main focus has been on those linear and nonlinear properties of collisionless trapped-electron modes which lead to a significant local current and/or $q$-dependence. Regarding linear characteristics, current profile dependencies are evident in Eq. (11) together with Figs. 1, 2, and 3. Prominent dependencies on $\hat{s}$ and $q$ are also found in the nonlinear results summarized in Eqs. (16), (18), (19), and (20). These finding have motivated ongoing studies ${ }^{16}$ to properly incorporate such effects into anomalous transport models used in interpreting experimental confinement results.

With regard to other collisonless electrostatic modes, non-resonant type microinstabilities such as the interchango-like "ubiquitous" trapped-electron modes, ${ }^{17}$ the trappedion interchange mode, ${ }^{18}$ and the toroidal $\eta_{i}$-mode ${ }^{19}$ can also exhibit a local scaling with q. For example, both the ubiquitous TEM and the collisionless TIM are characterized by linear growth rates scaling as $\gamma \propto\left(\omega_{* p} \bar{\omega}_{e d}^{(0)}\right)^{1 / 2} \propto G^{1 / 2}$. Also, Guo et al..$^{19}$ have indicated that the linear properties of toroidal $\eta_{i}$-modes in the weakly ballooning limit combined with heuristic $\gamma / k_{1}^{2}$-type diffusivity estimates can lead to $\chi_{i} \times[q(a)]^{3 / 2}$. In 
general, a strong turbulence type analysis is needed to properly assess the nonlinear consequences of all of these various types of nonresonant collisionless electrostatic instabilities.

Finally, in comparing predicted trends from theoretical models of electrostatic microinstabilites with experimental results, it would be very interesting to examine data which could relate changes in the local values of $q$ and $q^{\prime}$ in the bulk plasma region to observe confinement properties. It should also be kept in mind that when dealing with electrostatic turbulence, anomalous energy exchange between species could be non-neglibible for realistic comparisons of $\left|\chi_{e}\right|$ versus $\left|\chi_{i}\right|$ in power balance analyses of experimental data.

\section{Acknowledgment}

Work in this paper was carried out in collaboration with G. Rewoldt and T.S. Hahm. This research was supported by US Department of Energy contract number DE-AC0276-CHO3073. 


\section{References}

${ }^{1}$ F.W. Perkins, in Heating in Toroidal Plasmas (Proc. 4th. Int. Symp. Rome, 1984), Vod. 2, Int. School of Plasma Physics, Varenna (1984) 977.

${ }^{2}$ W.M. Tang, Nucl. Fusion 26, 1605 (1986).

${ }^{3}$ R.R. Dominguez and R.E. Waitz، Nucl. Fusion 27, 65 (1987).

${ }^{4}$ R.J. Goldston, Plasma Phys. Controlled Fusion, 26, 87 (1984).

${ }^{5}$ J.W. Connor and J.B. Taylor, Nucl. Fusion 17, 1047 (1977).

${ }^{6}$ W.K. Hagan and E.A. Frieman, Phys. Fluids 29, 3635 (1986).

${ }^{7}$ M.H. Redi, W.M. Tang, P.C. Efthimion, D.R. Mikkelsen, and G.L. Schmidt, Nucl. Fusion 27, 2001 (1987).

8J.C. Adam, W.M. Tang, and P.H. Rutherford, Phys. Fluids 19, 561 (1976).

${ }^{9}$ M.C. Zarnstorff, et al., in Proceedings of 16th. European Conference on Controlled Fusion and Plasma Heating, Vol. 1, 271 (1990).

${ }^{10}$ L. Chen, R.B. White, and M.N. Rosenbluth, Phys. Rev. Lett. 52, 1122 (1984).

${ }^{11}$ G. Rewoldt, W.M. Tang, and R.J. Hastie, Phys. Fluids 30, 807 (1987).

${ }^{12}$ T.S. Hahm and W.M. Tang, Princeton Plasma Physics Laboratory Report, PPPL-2721, September 1990; submitted to Phys. Fluids.

${ }^{13}$ R.Z. Sagdeev and A.A. Galeev, in Nonlinear Plasma Theory, edited by T.M. O'Neil and D.L. Book (Benjamin, New York, 1969).

${ }^{14}$ R.J. Goldston and Y. Takase (private communication).

${ }^{15}$ W. Horton, in Handbrook of Plasma Physics edited by M.N. Rosenbluth and R.Z. Sagdeev, Vol. 2, 383 (Elsevier, New York, 1984). 
${ }^{16}$ T.S. Hahm, S.C. Cowley, G.W. Hammett, R.M. Kulsrud, F.W. Perkins, M.H. Redi, G. Rewoldt, R.N. Sudan, and W.M. Tang, 13th. Int. Conf. on Plasma Phys. and Control. Nucl. Fus. Res'. Oct. 1990 (IAEA, Vienna, 1991), paper D-1-1.

${ }^{17}$ B. Coppi and G. Rewoldt, Phys. Lett. A 49, 36 (1974).

${ }^{18}$ B.B. Kadomtsev and O.P. Pogutse, Nucl. Fusion 11, 67 (1971).

${ }^{19}$ S. C. Guo, L. Chen, S.T. Tsai, and P.N. Guzdar, Plasma Phys. Controlled Fusion 31, 423 (1989). 

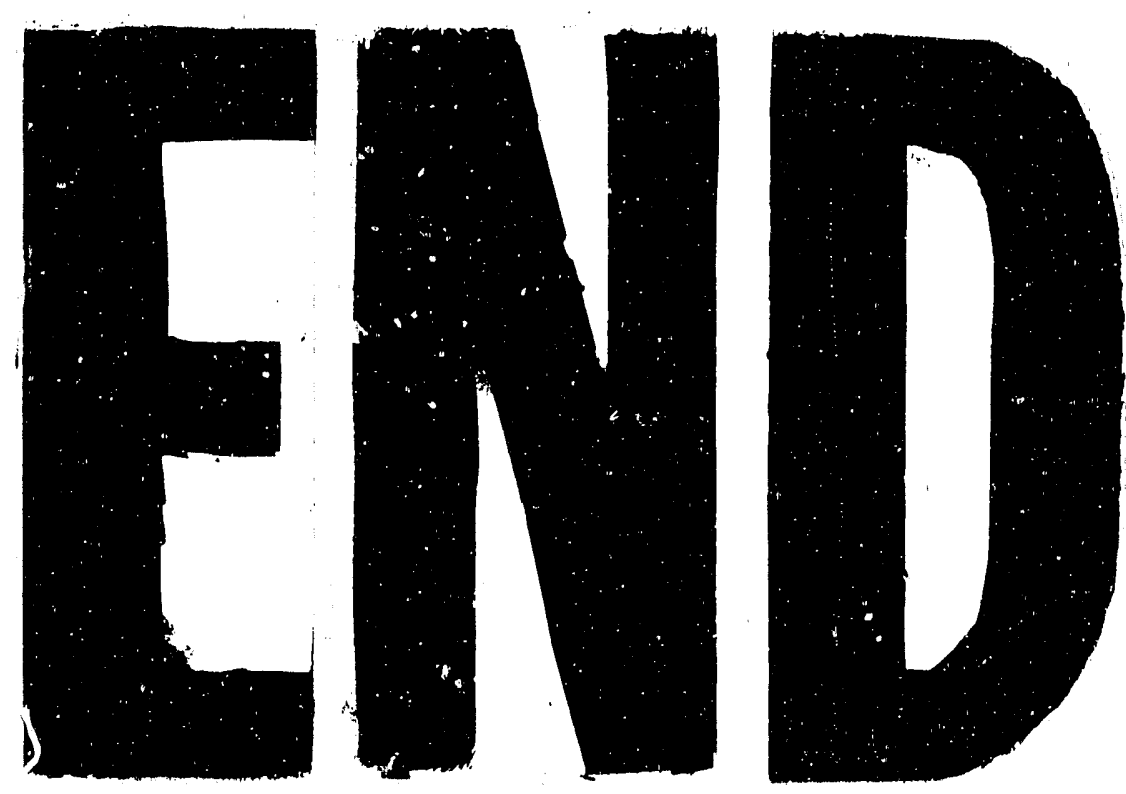

4
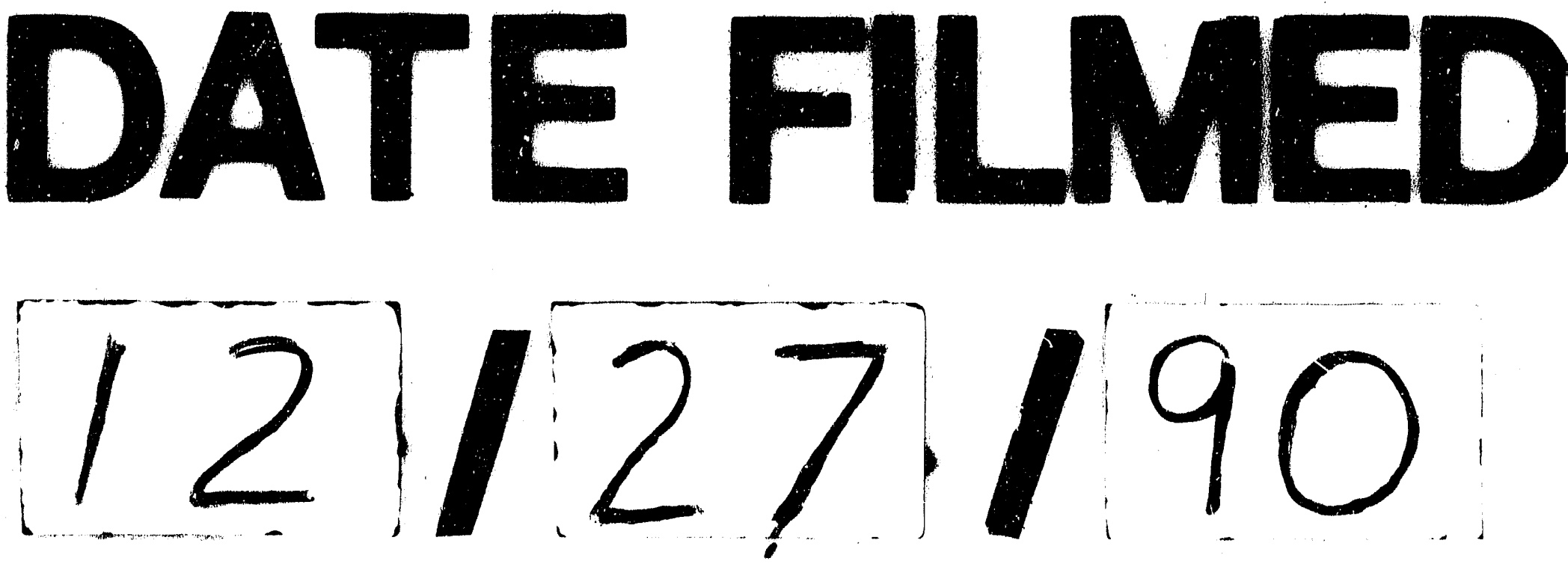
